If this problem is preceded by a full discussion of the following exercises, the class will find point $O$ without assistance:

(a) Every point in the common tangent to two equal tangent circles may be the center of a circle tangent to the two given circles.

(b) Every point in the common chord to two equal intersecting circles may be the center of a circle tangent to the two given circles.

(c) Every point in the perpendicular bisector of the line of centers of any two equal circles may be the center of a circle tangent to the two given circles.

(d) Every point in the circumference of a circle concentric with two given concentric circles and Iying half way between them may be the center of a circle tangent to the two given circies.

\title{
MORE COMMENTS.
}

By G. W. Greenwood, Dunbar, Pa.

In the January number of School Science and Mathematics, page 40 , it is suggested that "if a postulate leads to $* * *$ no fruitful scientific hypothesis" it has no real or objective meaning. It would of course be impossible to determine beforehand the value of a new and untried hypothesis, nor should we decide too hastily that a postulate is fruitless; negative numbers were at first called imaginary, or fictitious, and regarded merely as curiosities; complex variables, I believe, were found to be of real service in theoretical electricity; the pure study of conic sections, after centuries of comparative inutility, has become the corner stone of mathematical astronomy; "that a quantitive study of defective children should need the study first of the geometry of hyperspace may sound paradoxical, but it is none the less true"*; when Pasteur took up the subject of spontaneous generation his friends expressed their regret at his wasting energy on a "useless theory," yet upon the unforeseen results rest rast advances in medicine and surgery. Instances could be multiplied almost without number showing that only omniscience conld decide that any postulate was useless.

In fact, so prone are we to estimate relative values by proximate concrete returns that teachers should incline all the more strongly to emphasize the importance of research promising no immediate material rewards.

Again, whether in the election of a president or in the most trivial act calling for a conscious or unconscious exercise of reason, in making a postulate or a deduction from it, one's mental activity should be guided and strengthened by a proper study of geometry. Unfortunately, the subject as currently. presented is a most potent agency in dulling whatever reasoning powers the student may initially possess.

\footnotetext{
* "Grammar of Science," Karl Pearson. I am indebted for this quotation to Dr. $\mathrm{F}$. I. Reitz of the University of Illimois.
} 